Results Eight teams [ $\mathrm{n}=118$ players; 83 males, 35 females] consented to participate. All control teams and $3 / 4$ of the intervention teams completed all 6 sessions [median 85 days (range 42-102)]. No adverse events were reported. There were 6 concussions in the intervention group $(n=65)$ and 4 in the control group $(n=53)$. Exploratory univariate Poisson regression analysis adjusted for cluster by team revealed no difference in concussion risk between groups [incidence rate ratio $(\mathrm{IRR})=0.99$ concussions $/ 100$ players/season $(95 \% \mathrm{CI} ; 0.28-3.48)$. For males alone, there was also no difference in IRR $\left[\mathrm{IRR}_{\text {males }}=0.37(95 \% \mathrm{CI}\right.$; 0.068-1.94)]. Both female teams were allocated to the intervention group.

Conclusions The implementation of a neuromuscular and sensorimotor training program with youth ice hockey teams was feasible and safe. Future evaluation in a larger sample over a longer training period to examine the effects of neuromuscular and sensorimotor training strategies on the risk of concussion in youth ice hockey players, including consideration of sex and age group is warranted.

\section{THE EFFECT OF THE FÉDÉRATION INTERNATIONALE DE FOOTBALL ASSOCIATION (FIFA) 11+ KIDS INJURY PREVENTION PROGRAM ON REDUCING INJURY RATES IN CHILDREN'S SOCCER: A CLUSTER-RANDOMIZED CONTROLLED TRIAL}

\begin{abstract}
1,2,3 Wesam Saleh A Al Attar, ${ }^{4}$ Saud Alarifi, ${ }^{5}$ Hussain Saleh H Ghulam, ${ }^{6}$ Hosam Alzahrani, ${ }^{7}$ Msaad M Alzhrani, ${ }^{3}$ Ross H Sanders. 'Department of Physical Therapy, Faculty of Applied Medical Science, Umm Al Qura University, Makkah, Saudi Arabia; ${ }^{2}$ Department of Sport, Exercise and Health, Faculty of Medicine, University of Basel, Basel, Switzerland: ${ }^{3}$ Discipline of Exercise and Sport Science, Faculty of Medicine and Health, The University of Sydney, Sydney, Australia; ${ }^{4}$ Department of Physical Therapy, King Abdulaziz Medical City, Riyadh, Saudi Arabia; ${ }^{5}$ Department of Rehabilitation Medical Sciences, Faculty of Applied Medical Science, Najran University, Najran, Saudi Arabia; ${ }^{6}$ Department of Physiotherapy, College of Applied Medical Sciences, Taif University, Taif, Saudi Arabia; ${ }^{7}$ Department of Physical Therapy, Faculty of Applied Medical Science, Majmaah University, Majmaah, Saudi Arabia
\end{abstract}

\subsection{6/bjsports-2021-IOC.164}

Background The Fédération International de Football Association (FIFA) $11+$ Kids injury prevention program is an exercise-based program developed by an international group of experts to prevent injuries among children's soccer players.

Objective The objective of this study is to evaluate the effect of the FIFA $11+$ Kids injury prevention program on reducing the incidence of injuries among children soccer players aged 7-13 years.

Design A cluster-randomized controlled trial.

Setting Children's soccer.

Patients (or Participants) Ninety-four boys' soccer teams (780) players (under 8 years, under 9 years, under 11 years, and under 13 years age groups) were randomly allocated into the experimental or a control group.

Interventions (or Assessment of Risk Factors) The experimental group performed the FIFA $11+$ Kids injury prevention program as warm-up during training sessions for at least twice a week, and the control group performed their usual warm-ups. Participants were prospectively followed during one season.

Main Outcome Measurements The primary outcome was to investigate the incidence of initial, recurrent injury, mechanism of injury and injury severity.

Results A total of 43 injuries were reported among 391 players in the experimental group in 8353.33 hours of exposure
(0.85 injuries/1000 exposure hours), and a total of 86 injuries were reported among 389 players in the control group in 7102.67 hours of exposure (2.01 injuries/1000 exposure hours). The injury risk ratio IRR was 0.43 , which suggests that the injuries in the experimental group were $57 \%$ less in comparison to the control group.

Conclusions Implementation of the FIFA $11+$ Kids injury prevention program reduced overall injury rates in boys' soccer players more than the usual warm-up.

\section{THE THE FÉDÉRATION INTERNATIONALE DE FOOTBALL ASSOCIATION (FIFA) 11+ KIDS INJURY PREVENTION PROGRAM: AWARENESS, IMPLEMENTATION, AND OPINION OF WORLDWIDE CHILDREN'S SOCCER COACHES}

1,2,3 Wesam Saleh A Al Attar, 'Saad M Alhosaini, 'Sami Z Alharbi, 'Yasser M Alraddadi, ${ }^{4}$ Hussain Saleh H Ghulam, ${ }^{5}$ Mashaer Alyami, ${ }^{3}$ Ross H Sanders. 'Department of Physical Therapy, Faculty of Applied Medical Science, Umm Al Qura University, Makkah, Saudi Arabia; ${ }^{2}$ Department of Sport, Exercise and Health, Faculty of Medicine, University of Basel, Basel, Switzerland; ${ }^{3}$ Discipline of Exercise and Sport Science, Faculty of Medicine and Health, The University of Sydney, Sydney, Australia; ${ }^{4}$ Department of Rehabilitation Medical Sciences, Faculty of Applied Medical Science, Najran University, Najran, Saudi Arabia; ${ }^{5}$ King Fahad Specialist Hospital, Dammam, Saudi Arabia

\subsection{6/bjsports-2021-IOC.165}

Background The Fédération Internationale de Football Association (FIFA) has promoted the FIFA $11+$ injury prevention program worldwide. The FIFA $11+$ injury prevention program for kids was developed and shown to be effective in reducing soccer-related injuries in numerous studies.

Objective To assess the awareness, implementation, and opinion of worldwide children's soccer coaches regarding the FIFA $11+$ Kids injury prevention program.

Design Cross-sectional study.

Setting An online survey for all continental football federations. Patients (or Participants) A total of 486 children's soccer coaches completed the survey.

Interventions (or Assessment of Risk Factors) The questionnaire consisted of questions relating to awareness, implementation, and coaches' opinions of the FIFA $11+$ Kids injury prevention program.

Main Outcome Measurements The awareness level, implementation rate, and opinion of the the FIFA $11+$ Kids injury prevention program efficacy.

Results A total of 202 (41.6\%) of the children's soccer coaches reported awareness of the FIFA $11+$ Kids injury prevention program and 155 (31.9\%) were reported implementing the FIFA $11+$ Kids injury prevention program in their current practice. Participants who reported implementation of the FIFA $11+$ Kids injury prevention program also reported a positive attitude towards the program efficacy, with a score of $8.19 \pm 0.88$ out of 10 .

Conclusions Globally, children's soccer coaches showed poor awareness levels of the FIFA $11+$ Kids injury prevention program, however, the implementation level among coaches who reported awareness was relatively high. A high number of children's soccer coaches reported a positive perception regarding the efficacy of the FIFA $11+$ Kids injury prevention program in reducing and preventing injuries. Therefore, there is a need for further efforts and research to increase the international awareness and implementation rate of the FIFA $11+$ Kids injury prevention program. 